\section{Improved beam uniformity in multimode fibers using piezoelectric-based spatial mode scrambling for medical applications}

\author{
Lianshan Yan, ${ }^{a}$ X. Steve Yao, ${ }^{b}$ Lynn Lin, ${ }^{b}$ \\ and Xiaojun Chen ${ }^{b}$ \\ a Southwest Jiaotong University, Center for Information \\ Photonics \& Communications, Chengdu, Sichuan, \\ China, 610031 \\ ${ }^{\mathrm{b}}$ General Photonics Corp., 5228 Edison Avenue, Chino, \\ California 91710 \\ E-mail: Isyan@home.swjtu.edu.cn
}

\begin{abstract}
We improve the beam uniformity passing through multimode fibers using piezoelectric-based spatial mode scrambling. Both fiber squeezing and stretching techniques are applied and compared. A more than sixfold difference in the power intensity crossing the output beam profile without mode scrambling can be reduced to a $<10 \%$ variation. The efficiency of collecting useful optical power for detection is also significantly improved. Such modules can find various applications in medical imaging, disease diagnosis, and local area networks. () 2008 Society of Photo-Optical Instrumentation Engineers.

[DOI: 10.1117/1.2976419]
\end{abstract}

Subject terms: multimode fiber; spatial mode scrambling; optical beams; uniformity.

Paper 080231LR received Mar. 26, 2008; revised manuscript received Jul. 1, 2008; accepted for publication Jul. 2, 2008; published online Sep. 4, 2008.

\section{Introduction}

Having a uniformly distributed light beam is highly desirable for various applications where multimode fibers (MMFs) are used as the light transmission or guiding media. In addition to conventional applications, including optical local area networks (LANs) over MMF and image encryptions, recent advances in genetic analysis and functional genomics, such as massively parallel signature sequencing (MPSS) and molecular interactions, have made the practical and reliable requirements more and more critical. ${ }^{1-4}$

Mode scrambling has been a well-known technique to improve the uniformity in multimode fibers. So far, various approaches have been demonstrated: (i) modifying the multimode fiber itself, as through extremely high-temperature annealing ${ }^{5}$ or special fabrication in the cladding; ${ }^{6}$ (ii) twisted plastic optical fiber instead of traditional MMF; ${ }^{7}$ (iii) selecting one special mode through mode coupling. 8 These approaches suffer from one or more disadvantages, such as quality uncertainty or extreme manufacture requirements, higher cost of the fiber itself, or introducing considerable loss. Providing a more practical solution with longer lifetime and reliable performance is one of the major challenges for various mentioned applications.

0091-3286/2008/\$25.00 @ 2008 SPIE
In this letter, we demonstrate the improvement of beam uniformity through MMF using spatial mode scrambling. This is accomplished through either fiber squeezing or fiber stretching. Both approaches are based on the practical and well-qualified piezoelectric actuator technology. The output beam uniformity after the step-indexed MMF is analyzed and compared. The intensity fluctuations are reduced significantly; as an example, using the fiber squeezing technique, a more than sixfold relative (normalized) power intensity fluctuation without mode scrambling can be reduced to an almost uniform distribution ( $<10 \%$ variation).

\section{Configuration}

In order to improve the beam uniformity, we use two different schemes, as shown in Fig. 1, both based on piezoelectric actuator technology. Figure 1(a) uses fiber squeezing to generate spatial mode scrambling inside the optical fiber itself. Figure 1(b) shows a scheme that uses the piezoelectric actuator to stretch the MMF.

In Fig. 1(a), the mode scrambler consists of the multimode fiber itself (we use a step-index MMF) and six sections of fiber squeezers. These fiber squeezers add stress through piezoelectric actuators at different orientations on the multimode fiber, typically with a $45-\mathrm{deg}$ shift between adjacent sections $(0,45,0,45,0$, and 45 deg in the illustrated example). Driving signals are applied with different frequencies (or even amplitudes if necessary) to further randomize the mode coupling along the multimode fiber (mostly around $500 \mathrm{~Hz}$, with slight differences in our experiments). The amplitudes of the driving signals are all close to $150 \mathrm{~V}$, which is the limit of our driving circuits.

In Fig. 1(b) for the case using fiber stretching, a fiber coil (with a step-index MMF) is made on a fiber stretcher frame. Instead of applying stress to the fiber, the piezoelectric actuators (four are used) are driven to provide longitudinal extension at high frequencies $(\approx 2.4 \mathrm{kHz})$, thereby generating spatial mode scrambling along the MMF. Since the driving frequencies are near resonance frequencies of

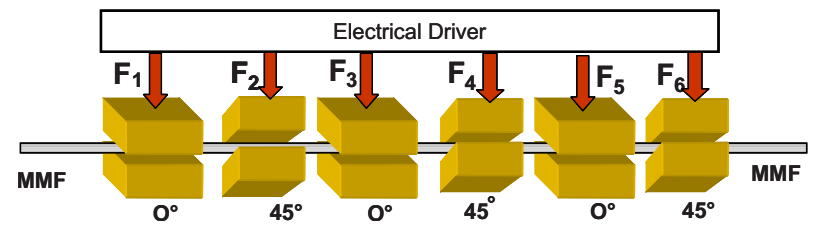

(a)

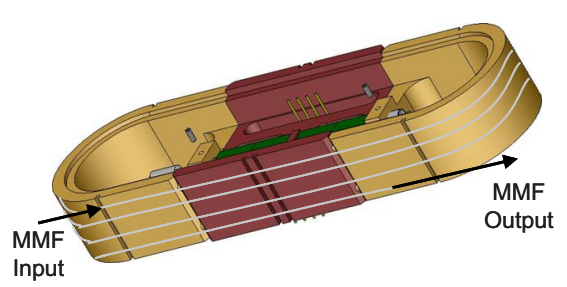

(b)

Fig. 1 Conceptual diagram of multimode fiber (MMF) mode scrambler: (a) using fiber-squeezing technology: six electrically driven fiber squeezers are applied along the MMF with different orientation between sections; (b) using fiber-stretching technique with MMF: four piezoelectric actuators are used to produce longitudinal extension. 


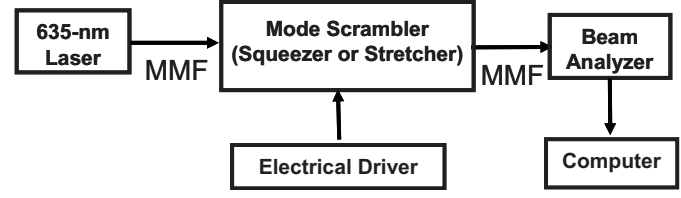

Fig. 2 Evaluation setup of the mode scrambler using either fiber squeezing or fiber stretching through MMF.

the actuators (the resonance window is several hundred hertz, not sensitive to frequency drift), the amplitudes of the applied signals are much less than in the fiber-squeezing approach (typically $\approx 30 \mathrm{~V}$ ), a potential advantage for practical applications.

\section{Experimental Results and Discussion}

To evaluate the performance of our approaches, we set up a testing stage (Fig. 2) where a beam analyzer is used to detect the output beam profile after the MMF. The wave- length of the light source is set to $635 \mathrm{~nm}$ (Thorlabs LPS635-FC fiber-coupled laser). Electrical signals are generated by our circuit and applied to piezodrivers to provide either fiber squeezing or stretching (six and four driving signals are used for the squeezing and the stretching, respectively). The aperture of the beam analyzer is set to $420 \mu \mathrm{m}$, and the final beam profile (data) is recorded in the computer, generally with an $80-\mathrm{ms}$ integration time. The insertion loss of the fiber-squeezing module is typically $<0.5 \mathrm{~dB}$ (including the connector loss); for the stretching modules it is $\approx 0.8 \mathrm{~dB}$

The final improvements are compared in Fig. 3. Figure $3(\mathrm{a})$ is the beam profile without mode scrambling, while Fig. 3(b) and 3(c) show the profile improvement using fiber squeezing and fiber stretching, respectively. The effectiveness of both approaches is abvious. The $X-Y$ beam analyses crossing the center of the output light are also displayed next to the beam profiles for each case. We note that the intensity fluctuation without mode scrambling is more than 6 times (e.g., between 0.15 and 1.0 after normalization);
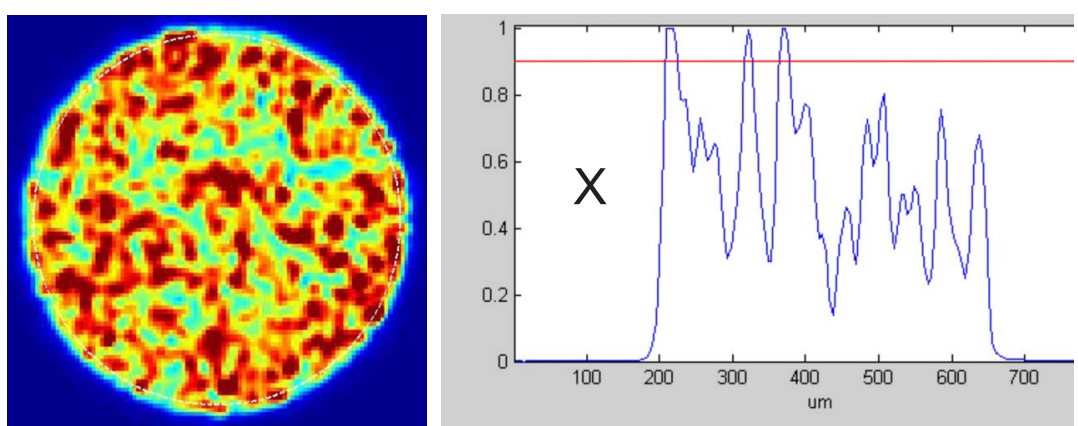

(a)
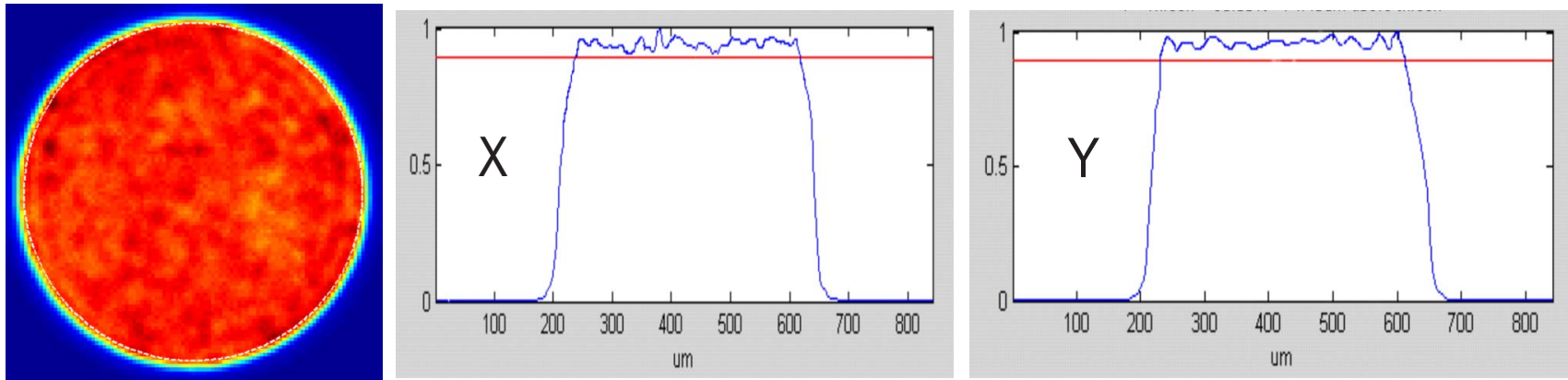

(b)
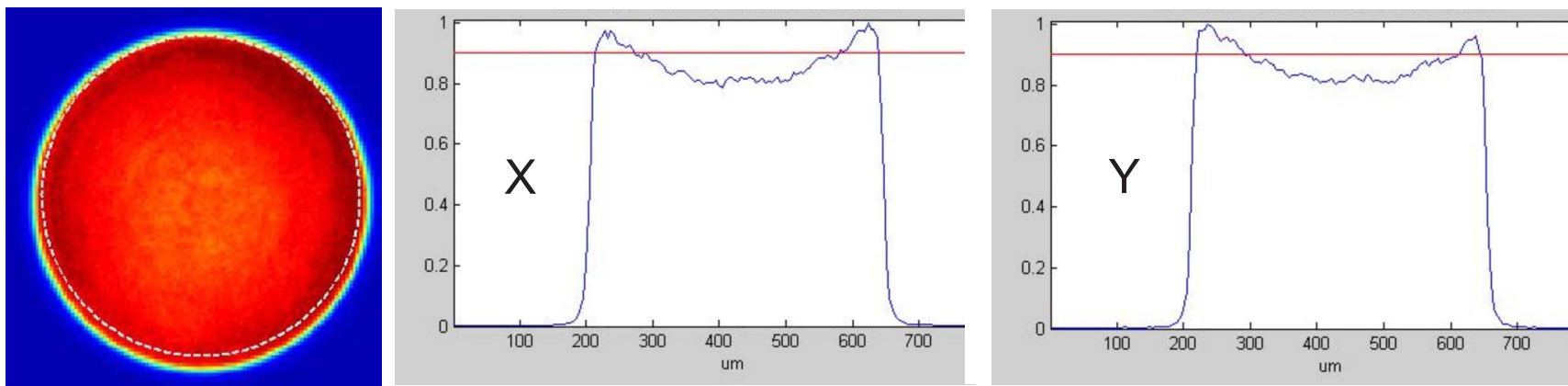

(c)

Fig. 3 Improvement of the light uniformity through mode scrambling (both traces $X$ and $Y$ lie at the center of the profile): (a) without scrambling, (b) using fiber-squeezing, (c) using fiber stretching. 
while with mode scrambling, the fluctuation is reduced to $<10 \%$ and $<20 \%$ for the fiber squeezing and stretching, respectively. In fact, the figures of merit of the spatial mode scrambling include not only the deviation of the beam uniformity, but also the efficiency of collecting more optical power into the useful detection area (for better illumination), because the light will diffuse more without mode scrambling. If we define the scrambling efficiency as the percentage of the effective optical power into the detection area, the corresponding efficiencies are $\approx 40 \%$ (without mode scrambling), $\approx 78 \%$ (with fiber stretching), and $\approx 84 \%$ (with fiber squeezing).

It is interesting to see that the beam uniformity using fiber squeezing is better than that using fiber stretching. We believe that this is mainly due to more random spatial scrambling along the six squeezing sections than with only one direction (i.e., longitudinal) of stretching. This is also a possible reason that a dip exists at the center of the power distribution [Fig. 3(c)] for the stretching case. On the other hand, because the fiber is much longer (tens of meters) and the piezoelectric actuators are driven at higher frequencies, the overall uniformity is still improved a lot through fiber stretching.

\section{Conclusion}

Significantly improved beam uniformity passing through MMF was realized through fiber squeezing or fiber stretch- ing. Such mode scramblers are key modules for various applications, including medical imaging, disease diagnosis, and local area networks.

\section{Acknowledgments}

The authors would like to thank Karen Li and Peter Betts at General Photonics Corp. for help in the measurements.

\section{References}

1. A. D. Mehta, J. T. Finer, and J. A. Spudich, "Detection of singlemolecule interactions using correlated thermal diffusion," Proc. Natl. Acad. Sci. U.S.A. 94, 7927-7931 (1997).

2. Y. T. Chen, M. J. Scanlan, C. A. Venditti, R. Chua, G. Theiler, B. J. Stevenson, C. Iseli, A. C. Gure, T. Vasicek, R. L. Strausberg, C. V. Jongeneed, L. J. Old, and A. J. G. Simpson, "Identification of cancer/ testis-antigen genes by massively parallel signature sequencing," Proc. Natl. Acad. Sci. U.S.A. 102(22), 7940-7945 (2005).

3. G. A. Stolovitzky, A. Kundaje, G. A. Held, K. H. Duggar, C. Haundenschild, D. Zhou, T. Vasichek, K. Smith, A. Aderem, and J. Roach, "Statistical analysis of MPSS measurements: application to the study of LPS-activated macrophage gene expression," Proc. Natl. Acad. Sci. U.S.A. 102(5), 1402-1407 (2005).

4. I. H. White, R. V. Penty, J. Hankey, K. A. Williams, G. F Roberts, M. Glick, and D. McAuley, "Optical local area networking using CWDM," Proc. SPIE 5248, 284-293 (2003)

5. J. B. Schlager and A. H. Rose, "Annealed optical fiber mode scrambler," Electron. Lett. 37(1), 9-10 (2001).

6. L. L. Blyler, L. G. Cohen, G. J. Grimes, and L. J. Haas, "Mode scrambler with noninvasive fabrication in an optical fiber's cladding," U.S. Patent No. 4,974,930 (1990).

7. J. Arrue, G. Aldabaldetreku, G. Durana, J. Zubia, I. Garces, and F. Jimenez, "Design of mode scramblers for step-index and gradedindex plastic optical fibers," J. Lightwave Technol. 23(3), 1253-1260 (2005).

8. J. P. Bristow and J. A. Lehman, "Fiber optic mode scrambler," U.S Patent No. 5,892,866 (1999). 